\title{
NUEVAS TECNOLOGÍAS EN LOS CONTROLADORES DIGITALES DE SEÑALES, ESTUDIO Y APLICACIONES
}

\section{NEW TECHNOLOGY IN DIGITAL SIGNAL CONTROLLERS, STUDY \& APPLICATIONS}

\section{Jaime Fonseca-Beltrán1; Gerardo Cazarez-Ayala1; Flor Kalíope Lomeli Dablantes2}

Profesor Investigador Instituto Tecnológico de Los Mochis, Departamento de Ingeniería Eléctrica y Electrónica, Blvd. Juan de Dios Batiz y 20 de Noviembre, Los Mochis, Sinaloa1. Residente Profesional, Instituto Tecnológico de Los Mochis2.

\section{RESUMEN}

En el presente trabajo se abordan las nuevas tecnologías de los controladores digitales de señales (DSC), se hace una referencia general de las diferentes compañías fabricantes de este tipo de dispositivo, con el propósito que el lector tenga una idea generalizada de los diferentes productos existentes en el mercado, resaltando en ello las diferencias existentes con sus antecesores, de tal forma que sea posible identificar de una manera clara y precisa tales diferencias.

El presente trabajo fue concebido con la finalidad de facilitar al usuario, una transición en el manejo de microncontroladores PICs ${ }^{\circledR}$ de proposito general, hacia el manejo de los microcontroladores avanzados como son los dsPIC., mismos que se pueden encontrar desde los uso de propósito general, hasta los que cuentan características especiales encaminadas hacia aplicaciones mas especializadas.

Palabra clave: Controlador Digital de Señal, Microcontrolador, dsPIC®.

\section{SUMMARY}

This paper addresses the new technologies of digital signal controllers (DSC), is a general reference of the different companies Recibido: 04 de diciembre de 2011. Aceptado: 02 de marzo de 2012. Publicado como ARTÍCULO CIENTÍFICO en Ra Ximhai 8(3): $247-254$ making this type of device, in order that the reader has a general idea of the various products available market, highlighting the differences it with its predecessors, so as to identify in a clear and precise differences.

This work was conceived in order to provide the user, a transition in the management of PIC (B) microcontrollers general purpose, to the management of advanced microcontrollers such as the dsPIC., Same to be found from the use of general purpose, even those who have special features designed to more specialized applications.

Keyword: Digital Signal Controller Microcontroller, dsPIC®.

\section{INTRODUCCIÓN}

Los Controladores Digitales de Señal (DSC) surgieron paramanejarmuchos de los desafíos que la ingeniería requiere actualmente, integrar a los microcontroladores (MCU) y Procesadores Digitales de Señal (DSP).

Como los microcontroladores, los DSC tienen respuestas rápidas de interrupción, periféricos orientados al control como son los PWM y watchdog timers. Por el lado de los DSP, éstos incorporan características tales como, en un solo ciclo multiplica y 
acumula (MAC), barrel shifter y grandes acumuladores.

Actualmente, los DSC se comercializan como las tecnologías verdes por su potencial para reducir el consumo de energía en motores eléctricos y fuentes de alimentación. Las tres principales compañías comercializadores de DSC son Texas Instruments, Freescale y Microchip Technology Inc. Estas tres empresas dominan el mercado de los DSC, con otros proveedores como Infineon y Renesas.

Microchip con sus familias dsPIC30F y dsPIC33F ofrecen opciones de sistemas de bajo costo y consumo de energía, alto rendimiento, aplicaciones de propósito general, control de motores, conversión de potencia, control de sensores, por mencionar algunas, una arquitectura Harvard modificada con un conjunto de instrucciones mejorado, pueden alcanzar hasta 40 MIPS. Texas Instruments ofrece alto rendimiento, ultra bajo consumo de energía, una arquitectura supersegmentada, además de alcanzar hasta 150 MIPS y una amplia gama de aplicaciones. Por otro lado, las familias de DSC de Freescale ofrecen opciones optimizadas para la conversión digital de potencia, control de motores y aplicaciones que requieran de alta velocidad, combinando la funcionalidad de los microcontroladores con un conjunto flexible de periféricos, creando soluciones de bajo costo.

Un dsPIC ${ }^{\circledR}$ es la adición de un microcontrolador de alto rendimiento de 16 bit con la capacidad de un DSP que se distingue por ser rápido, tener un manejo de interrupciones sofisticada y flexible, una amplia gama de funciones periféricas digitales y analógicas, de administración de energía, opciones flexibles de reloj, encendido (Power On Reset), protección contra caídas de tensión (brown-out), watchdog timer, código de seguridad y emulación de alta velocidad en tiempo real.

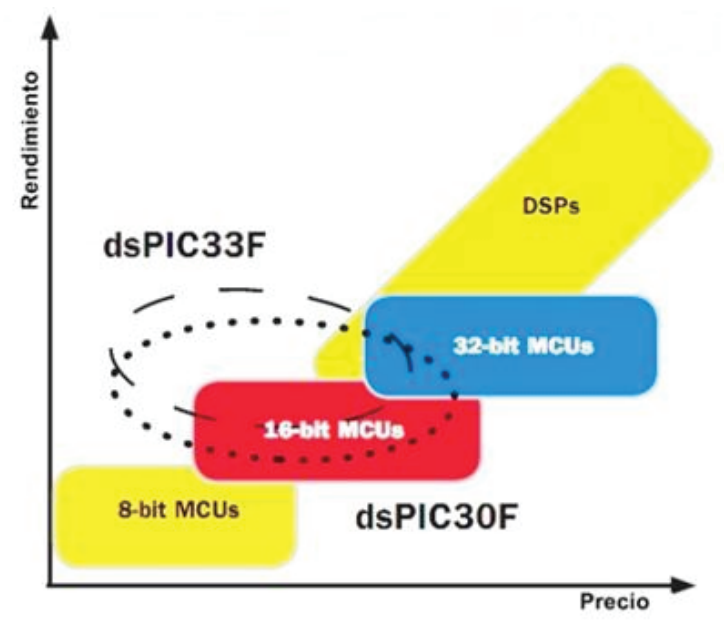

Figura 1. Las familias de dsPIC incluyen las principales prestaciones de los DSP y MCU de 16 bit y 32 bit.

\section{MATERIALES Y MÉTODOS}

El desarrollo del estudio se realizo en el Laboratorio de Electrónica del Instituto Tecnológico de Los Mochis, ya cuanta con la infraestructura (instalaciones, conectividad, equipo y materiales) necesaria para la implementación y prueba de aplicaciones basadas en los DSC. (www.itmochis.edu. $\mathrm{mx}$ ).

En primera instancia se realizo un estudio de los diferentes fabricantes de DSC a nivel mundial, de tal manera que nos dio un panorama general de la tendencia actual de tales dispositivos. Una vez que se conto con la información suficiente se determino trabajar con los DSC de la compañía Microchip Technology Inc, ya que sus dispositivos cubren todas las necesidades para el desarrollo de nuestras aplicaciones, sumado a ello la facilidad que se tiene al 
acceso de los dsPIC y al soporte que prestan para el desarrollo de aplicaciones basadas en esos dispositivos.

\section{RESULTADOS}

Una vez realizada la investigación documental se pueden mencionar algunas de las principales diferencias entre un DSP, un MCU y un DSC:

- Los DSP usan arquitectura Hardvard, que proporciona una memoria de datos separada de la memoria de programa. Los dos buses trabajan simultáneamente. Además incluyen Multiplicación por hardware, Multiplicación y Acumulación en un sólo ciclo de reloj, eficiencia en cálculo, requieren menos memoria, lenguaje ensamblador tipo algebraico y pueden combinar muchas características de control avanzadas.

- En cambio, los MCU usan arquitectura Von Neumann, la cual tiene el mismo espacio para la memoria de datos y programa. Las desventajas frente a los DSP incluyen la multiplicación en varios ciclos, solo algunos microcontroladores tienen multiplicador por hardware, necesitan muchas más memoria y el lenguaje ensamblador está basado en nemotécnicos.

- Por lo contrario, los DSC combinan el alto rendimiento del procesamiento de señal junto con las eficientes capacidades del control de procesos, además, ofrecen los mismos periféricos que los microcontroladores (Ethernet, USB, UART, SPI, I2C, ADC, CAN, etc.), cuentan con control de consumo de energía, encapsulados pequeños y son de bajo costo. A continuación se realiza una comparación entre las familias dsPIC30 y la dsPIC33, las cuales son el tema toral de nuestra investigación.
DIFERENCIAS ENTRE LAS FAMILIAS dsPIC30F Y dsPIC33F

Aunque las familias de dsPIC30F y dsPIC33F son muy similares, existen algunas diferencias entre ambos DSC, las cuales se presentan en el cuadro 1 .

Los dsPIC30F tienen como voltaje nominal 5 $\mathrm{V}$ y los dsPIC33F 3.3 V. También difieren en los pines de $\mathrm{I} / \mathrm{O}$ (Entrada/Salida), la memoria de programa (Flash) alcanza 144KB en los dsPIC30F y 256KB en los dsPIC33F. Los dsPIC33F carecen de EEPROM pero su SRAM puede llegar a $30 \mathrm{~KB}$ mientras que los dsPIC30F llegan hasta 8KB. Los dsPIC33F disponen del doble de interruptores y un controlador DMA. El rendimiento de los dsPIC30F es de 30 MIPS, en cambio, el dsPIC33F tiene un rendimiento de 40 MIPS. Ambos dsPIC cuentan con modos para controlar el consumo de energía como los son IDLE (modo de bajo consumo de energía) y SLEEP (modo de detección de voltaje bajo), el dsPIC33F cuenta con un modo extra denominado DOZEN el cual es un modo de frecuencia más baja.

Se puede encontrar una gran variedad de periféricos en las dos familias dsPIC ${ }^{\circledR}$ como temporizadores, convertidores $\mathrm{A} / \mathrm{D}$, módulos de captura y comparación, módulos PWM para el control de motores, módulos de comunicación SPI, CAN, UART, DCI, etc. Los dsPIC33F además contienen un Controlador de DMA y módulo de comunicación ECAN. También disponen de potentes herramientas para la gestión del sistema (Perro Guardián - Watch Dog), monitor de fallo de reloj, temporizadores para la estabilización del voltaje de alimentación y la frecuencia, etc.

\section{APLICACIONES}

A continuación, se presentan algunas las posibles aplicaciones de los DSC, 


\section{Cuadro 1. Principales diferencias entres} dsPIC30F y dsPIC33F

\begin{tabular}{|c|c|c|}
\hline Parámetros & $\begin{array}{l}\text { dsPIC30 } \\
\text { F }\end{array}$ & $\begin{array}{l}\text { dsPIC33 } \\
\text { F }\end{array}$ \\
\hline Modelos disponibles & 24 & 76 \\
\hline Arquitectura & 16 bit & 16 bit \\
\hline Velocidad CPU & 30 MIPS & 40 MIPS \\
\hline Tipo de memoria & Flash & Flash \\
\hline $\begin{array}{l}\text { Memoria } \\
\text { programa }\end{array}$ & $144 \mathrm{~KB}$ & $256 \mathrm{~KB}$ \\
\hline SRAM & $\begin{array}{l}8192 \\
\text { Bytes } \\
\end{array}$ & $\begin{array}{l}30720 \\
\text { Bytes }\end{array}$ \\
\hline $\begin{array}{ll}\text { Rango } & \text { de } \\
\text { temperatura } & \end{array}$ & $\begin{array}{l}-40-125 \\
{ }^{\circ} \mathrm{C}\end{array}$ & $\begin{array}{l}-40-150 \\
{ }^{\circ} \mathrm{C}\end{array}$ \\
\hline $\begin{array}{l}\text { Rango de voltaje de } \\
\text { operación }\end{array}$ & $\begin{array}{l}2.5-5.5 \\
\mathrm{~V}\end{array}$ & $3-3.6 \mathrm{~V}$ \\
\hline Pines E/S & 68 & 85 \\
\hline Número de pines & 80 & 100 \\
\hline $\begin{array}{l}\text { Características de } \\
\text { gestión del Sistema }\end{array}$ & $\begin{array}{l}\text { PBOR, } \\
\text { LVD }\end{array}$ & PBOR \\
\hline POR & $\mathrm{Si}$ & $\mathrm{Si}$ \\
\hline WDT & $\mathrm{Si}$ & $\mathrm{Si}$ \\
\hline Oscilador interno & $\begin{array}{l}7.37 \\
\mathrm{MHz}, \\
512 \mathrm{kHz}\end{array}$ & $\begin{array}{l}7.37 \\
\mathrm{MHz}, \\
512 \mathrm{kHz}\end{array}$ \\
\hline $\begin{array}{l}\text { Comunicación } \\
\text { periférica digital }\end{array}$ & $\begin{array}{ll} & 2 \\
\text { UART } & \\
\bullet & 2 \\
\text { SPI } & \\
\bullet & 1 \\
\text { I }^{2} \mathrm{C} & \end{array}$ & $\begin{array}{ll} & 2 \\
\text { UART } & \\
\bullet & 2 \\
\text { SPI } & \\
\bullet & 2 \\
\text { I }^{2} \mathrm{C} & \end{array}$ \\
\hline Periféricos Análogos & $\begin{array}{l}1-\mathrm{A} / \mathrm{D} \\
16 \mathrm{x} 10 \text { bit } \\
@ \quad 200 \\
(\mathrm{ksps})\end{array}$ & $\begin{array}{l}2-\mathrm{A} / \mathrm{D} \\
24 \times 12 \text { bit } \\
@, 500 \\
(\mathrm{ksps})\end{array}$ \\
\hline Comparadores & 4 & 4 \\
\hline CAN & $2 \mathrm{CAN}$ & 2 ECAN \\
\hline $\begin{array}{l}\text { Periféricos } \\
\text { Captura/Compara/P } \\
\text { WM }\end{array}$ & $8 / 8$ & $8 / 8$ \\
\hline $\begin{array}{l}\text { Canales de control de } \\
\text { motor PWM }\end{array}$ & 8 & 16 \\
\hline $\begin{array}{l}\text { Quadrature Encoder } \\
\text { Interface (QEI) }\end{array}$ & 1 & 1 \\
\hline Temporizadores & $\begin{array}{l}\left.\cdot{ }^{(16} \text { bit }\right)^{5} \\
\dot{(32}^{2}{ }^{2}\end{array}$ & $\begin{array}{l}\bullet \quad 9 \\
\left(16 \text { bit }{ }^{2}\right. \\
\text { (32 bit })^{2} 4\end{array}$ \\
\hline Puerto Paralelo & GPIO & GPIO \\
\hline RTCC & $\mathrm{Si}$ & $\mathrm{Si}$ \\
\hline DMA & 0 & 8 \\
\hline
\end{tabular}

clasificadas en las siguientes categorías:

A. CONTROL DE MOTORES

- Bombas industriales.

- Lavadoras y aspiradoras.

- $\quad$ Equipos de gimnasia.

- $\quad$ Tensores de cinturones de seguridad.

- Calefacción, ventilación y aire acondicionado

- Refrigeración.

- Herramientas eléctricas.

- $\quad$ Apertura de puertas.

- Control de estabilidad.

B. CONTROL DE SENSORES

- De torsión.

- De presión.

- De vibración.

- De humedad.

- De golpes.

- De lluvia.

- De fallos en la red eléctrica.

- De rotura de cristales.

- Químicos y de gases.

- $\quad$ Detección PIR avanzada 2-D.

C. AUTOMOCIÓN

- Control de potencia.

- Control de estabilidad y balanceo.

- Caja de cambios y embrague.

- Dirección hidráulica asistida

- Bolsa de aire (Airbag).

- Control de ignición.

- $\quad$ Sensor de presencia de ocupantes.

- $\quad$ Control del combustible.

D. CONECTIVIDAD EN INTERNET

- Monitorización remota.

- Contadores automáticos.

- Instrumentación médica remota.

- $\quad$ Sistemas de seguridad.

- Máquinas expendedoras.

- Diagnóstico industrial remoto.

E. AUDIO

- Reconocimiento de voz. 
- $\quad$ Altavoces telefónicos.

- Redes distribuidas de megafonía.

- $\quad$ Efectos especiales en instrumentos musicales.

- $\quad$ Cancelación de ruidos.

- Reproducción de sonido digital.

- Auriculares para la eliminación de ruidos.

- $\quad$ Puertos de comunicación para manos libres.

- $\quad$ Micrófono activado por la voz.

\section{dsPIC30F}

La familia de dispositivos dsPIC30F emplea una poderosa arquitectura Harvard modificada de 16 bits con un conjunto de instrucciones mejorado, para aplicaciones que dependen de alta velocidad, cálculos repetitivos, así como también, de control.

- El motor DSP, cuenta con dos acumuladores de 40 bits y soporte de hardware para las operaciones de división, barrel shifter, multiplicador de 17 x 17 bits, un banco de registros de trabajo de 16 bits y una amplia variedad de modos de direccionamiento de datos, todo en conjunto proporciona a la CPU una amplia capacidad de procesamiento matemático.

Microchip ha dividido los diferentes modelos de la serie dsPIC30F en tres familias caracterizadas por su área de aplicación, las

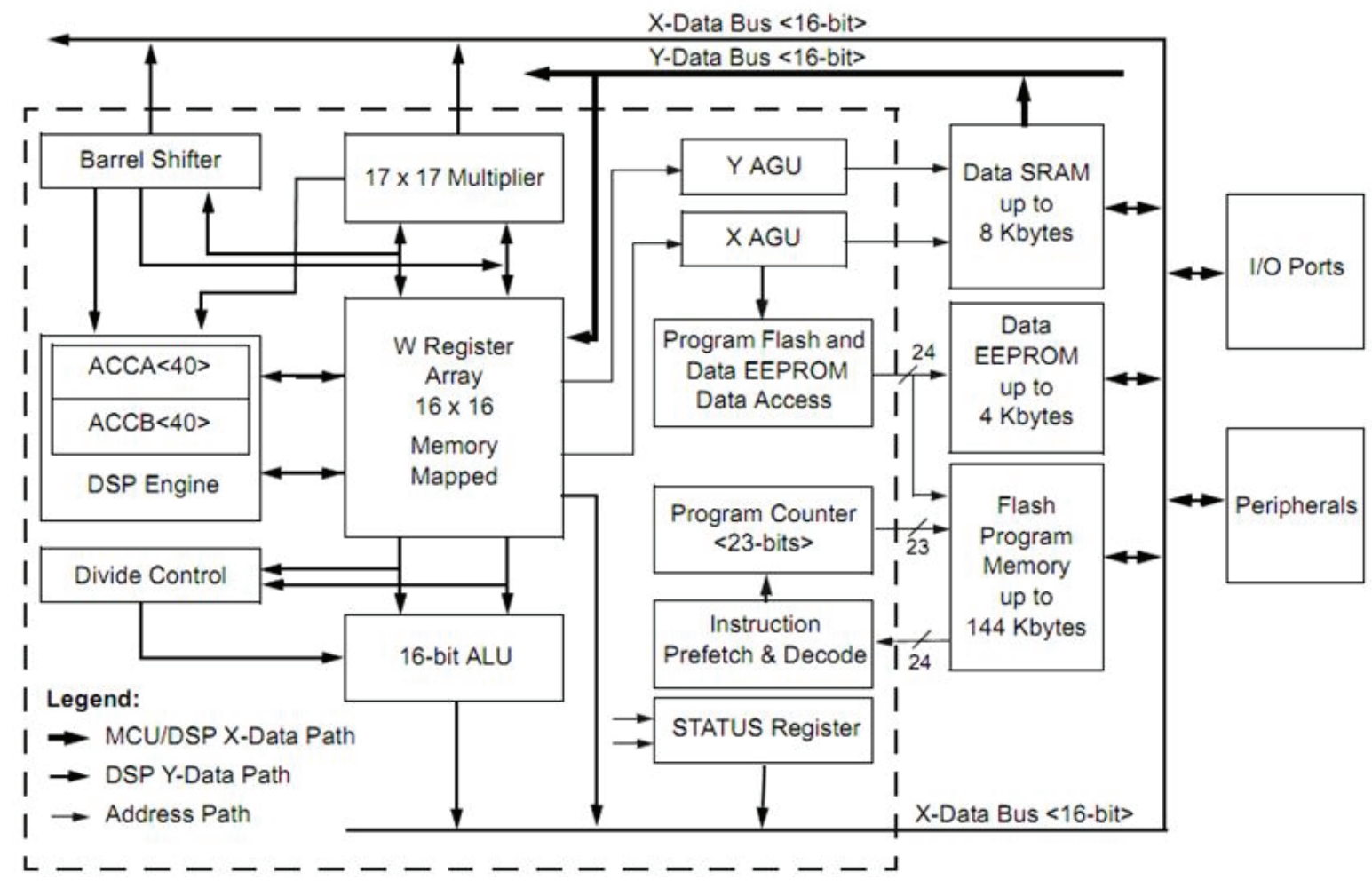

Figura 2. Diagrama a bloques de la familia de dispositivos dsPIC30F

que integra las características de control de un microcontrolador (MCU) con las capacidades de computacionales de un DSP. La funcionalidad resultante es ideal cuales son las siguientes:

- $\quad$ Familia de Propósito General.

- Familia de Control de Motores y Conversión de Potencia. 
- $\quad$ Familia de Control de Sensores.

\section{CARACTERÍSTICAS dsPIC30F}

El voltaje de alimentación admite un rango comprendido entre 2.5 y $5.5 \mathrm{~V}$. Se tolera una temperatura interna entre $-40^{\circ}$ y $85^{\circ} \mathrm{C}$ y una externa entre $-40^{\circ}$ y $125^{\circ} \mathrm{C}$. El rendimiento alcanza los 30 MIPS cuando el voltaje de alimentación tiene un valor entre 4.5 y 5.5 V.

En cuanto a la arquitectura de la CPU los dsPIC30F se sustentan en un núcleo RISC con arquitectura Harvard mejorada. Actuando como soporte central de información un banco de 16 registros de 16 bits cada uno; cuenta con un bus de datos de 16 líneas y otro de instrucciones de 24. Para potenciar la velocidad de las operaciones aritméticas complejas existe un Motor DSP que contiene un multiplicador de 17 x 17 bits, dos acumuladores de 40 bits y un robusto registro de desplazamiento. La memoria de programa, tipo FLASH, puede alcanzar un tamaño de $4 \mathrm{M}$ instrucciones de 24 bits cada una, aunque actualmente sólo hay modelos con una capacidad máxima de 256 $\mathrm{Kb}$. La memoria de datos se divide en dos espacios, X e Y, que pueden ser accedidos simultáneamente en las operaciones matemáticas DSP. Toda esta estructura admite operaciones MCU y operaciones DSP con un repertorio de 84 instrucciones.

Las secciones MCU y DSP cooperan en el funcionamiento general y comparten el flujo de instrucciones de los DSC. Los recursos específicos del Motor DSP, además de soportar las operaciones DSP, permiten implementar nuevas y potentes instrucciones MCU. Para reducir el tiempo de ejecución de algunas instrucciones DSP la memoria de datos SRAM se divide en dos espacios X e Y que pueden ser accedidos a la vez.

Otra característica importante en los

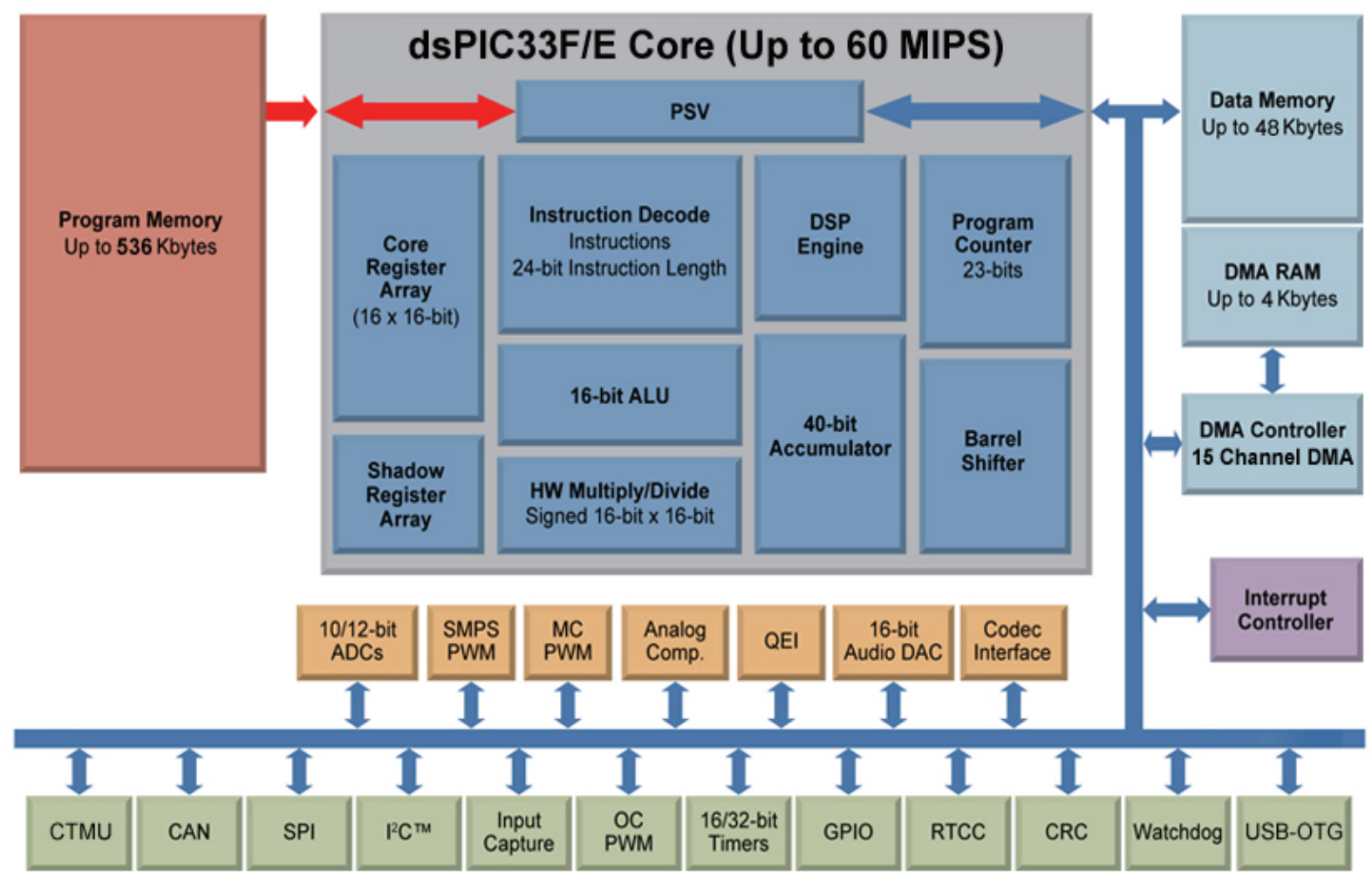

Figura 3. Diagrama a bloques general de la arquitectura del dsPIC33F. 
dsPIC30F es la de admitir hasta 45 fuentes distintas de petición de interrupción con 7 niveles de prioridad, de las cuales 5 son externas. Hay modelos de dsPIC30F que disponen de hasta 68 pines de E/S programables $\mathrm{y}$ con otras funciones multiplexadas con un consumo de $25 \mathrm{~mA}$ cada una.

dsPIC33F/E: DSC de alto desempeño

Los Controladores Digitales de Señal dsPIC33F ofrecen el rendimiento de un DSP con la simplicidad de un MCU. El núcleo del dsPIC33F está diseñado para ejecutar algoritmos de filtro digital de alta velocidad y precisión de los bucles de control digital, audio digital y procesamiento de voz.

Los dsPIC33F destinados al Control de Motores cuentan con periféricos que permiten el diseño de alto rendimiento, como lo son, los sistemas de control de la precisión del motor y fuentes de alimentación digital. Los periféricos SMPS son de alta velocidad y alta resolución PWM, rápidos ADC y comparadores analógicos.

Algunas de las características principales de los dsPIC33F, son las siguientes:

- $\quad$ Arriba de 60 MIPS a $3.3 \mathrm{~V}$.

- $\quad$ Un solo ciclo MAC 16x16.

- $\quad$ Acumuladores de 40 bits.

- Rápido DMA.

- Control de motor altamente integrado.

La familia de DSC dsPIC33F, emplea una poderosa arquitectura de 16-bit que integra las características de control de un microcontrolador (MCU) con el capacidades computacionales de un Procesador Digital de Señal (DSP). La funcionalidad resultante es ideal para aplicaciones que dependen de alta velocidad, cálculos repetitivos, así como de control. La serie de dispositivos dsPIC33F se divide en dos familias caracterizadas

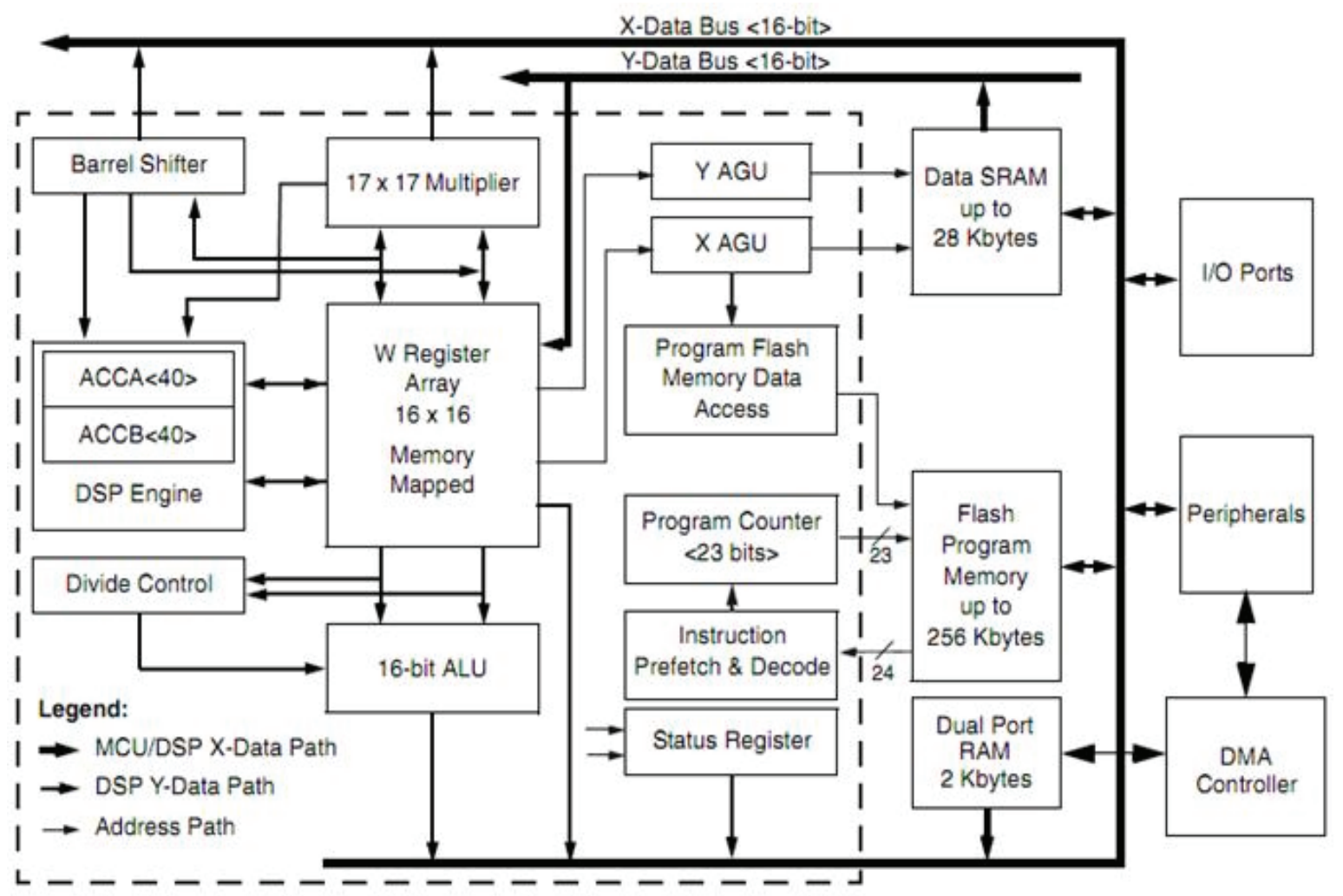

Figura 4. Diagrama a bloques de un dispositivo dsPIC33F 
por su área de aplicación, las cuales son las siguientes:

- $\quad$ Familia de Propósito General.

- $\quad$ Familia de Control de Motores.

El motor DSP, los dobles acumuladores de 40 bits, el soporte de hardware para operaciones de división, el multiplicador de $17 \times 17$, un gran conjunto de registros de trabajo de 16 bits y una amplia variedad de modos de direccionamiento de datos conforman la CPU del dsPIC33F con una extensa capacidad de procesamiento matemático.

El manejo de interrupciones flexibles y deterministas junto con una amplia variedad de periféricos hace que los dispositivos dsPIC33F sean adecuados para aplicaciones de control. Además, la Memoria de Acceso Directo (DMA) permite la transferencia de datos entre varios periféricos y una RAM dedicada DMA. La figura muestra un diagrama a bloques típico de un dispositivo dsPIC33F.

\section{CONCLUSIONES}

El incremento vertiginoso que se ha dado en el desarrollo de nuevas tecnologías para el procesamiento digital de señales, aunado al crecimiento que presenta los dispositivos móviles con procesamientos de audio, video y datos, ha llevado a que otras compañías fabricante de procesadores quieran incursionar en el diseño y fabricaciones de tales dispositivos, lo que sin duda traerá como beneficio en primera instancia, que se cuente con una gran variedad de DSCs tanto de propósito general, como aquellos de aplicaciones especificas. Por otro lado, sin duda alguna esta situación se reflejara en un decremento sustantivo en los costos de dichos dispositivos.
Por ello la importancia de realizar un estudio de los nuevos DSC existentes, y que sirva como pauta para el diseño e implementación de nuevas aplicaciones que ayude en la solución de problemas que se presentan en nuestro entorno, o en su defecto, que mejore los sistemas ya existentes.

\section{LITERATURA CITADA}

Angulo Usategui José María. dsPIC Diseño Prácticod e Aplicaciones. McGraw Hill,

Angulo Usategui José María. 2006. Microcontroladores Avanzados dsPIC. Thomson Learning.

Creed Huddleston. 2007, Intelligent Sensor Design using dsPIC, Elsevier Inc. www.microchip.com, www.ti.com, www.freescale.com

Jaime Fonseca Beltrán, Profesor Investigador Instituto Tecnológico de Los Mochis, Departamento de Ingeniería Eléctrica y Electrónica, Blvd. Juan de Dios Batiz y 20 de Noviembre s/n, C.P. 81279, Los Mochis, Sinaloa, México.

Gerardo Cázarez Ayala, Profesor Investigador Instituto Tecnológico de Los Mochis, Departamento de Ingeniería Eléctrica y Electrónica, Blvd. Juan de Dios Batiz y 20 de Noviembre s/n, C.P. 81279, Los Mochis, Sinaloa, México.

Flor Kaliope Lomelí Dablantes, Residente Profesional de la carrera de Ingeniería Electrónica, Instituto Tecnológico de Los Mochis, Blvd. Juan de Dios Batiz y 20 de Noviembre s/n, C.P. 81279, Los Mochis, Sinaloa, México. 